\title{
Measurement of Signal-to-Noise Ratio in Straw Tube Detectors for the PANDA Forward Tracker
}

Narendra Rathod, Jerzy Smyrski and Akshay Malige

\section{Self-Supporting Straw Tube Detectors}

The PANDA experiment [1] will be built at the FAIR facility in Darmstadt (Germany) to conduct experimental studies of the strong interaction through $\bar{p} p$ and $\bar{p} \mathrm{~A}$ annihilation. To track charged particles emitted at the most forward angles within acceptance of the PANDA Forward Spectrometer, the Forward Tracker (FT) consisting of a set of planar straw tube layers will be used [2]. The straws for the FT have a diameter of $10 \mathrm{~mm}$ and are made of $27 \mu \mathrm{m}$ thick aluminized Mylar foil [3]. As an anode, gold-plated tungsten-rhenium wire with $20 \mu \mathrm{m}$ diameter, is used. The straws are made self-supporting by means of 1 bar overpressure of the working gas mixture - $\mathrm{Ar} / \mathrm{CO}_{2}$ (90:10). The straws are read out by Front-End Electronics (FEE) cards based on the PASTTREC chip [4].

\section{Signal-to-Noise Ratio}

The signal-to-noise ratio was measured for the straw tube pulses at the analog output of the FEE card. The pulses were registered using CAEN Digitizer DT5742 working at a sampling frequency $1 \mathrm{GHz}$ and a resolution of $0.25 \mathrm{mV}$. Measurements were done for the straw illuminated with $5.9 \mathrm{keV} \mathrm{X}$-rays from ${ }^{55} \mathrm{Fe}$ source, at the anode voltage of $1700 \mathrm{~V}$. At this voltage, the straw pulses have an amplitude close to one observed for minimum ionizing protons crossing the straw in the vicinity of the anode wire and the anode voltage set to the foreseen working voltage of $1800 \mathrm{~V}$. The measurements were done for the gain in the FEE set to $1 \mathrm{mV} / \mathrm{fC}$ and three different settings of the peaking time parameter: 15, 20 and $35 \mathrm{~ns}$.

Narendra Rathod, Jerzy Smyrski and Akshay Malige

The Marian Smoluchowski Institute of Physics, Jagiellonian University, Łojasiewicza 11, 30-348

Kraków, Poland, e-mail: nsrathore.rajput@gmail.com 
The average amplitude of pulses corresponding to absorption of the $5.9 \mathrm{keV} \mathrm{X}$-rays from ${ }^{55} \mathrm{Fe}$ was determined from a gaussian function fit to the amplitude spectrum (see Fig. 1). For each registered waveform, the baseline level was determined as an average of the first 100 samples preceding the straw pulse and then was subtracted from all samples in the waveform. A distribution of the first 100 samples corrected for the baseline was fitted with a gaussian function and the standard deviation of the function was taken as the level of noise (see Fig. 2). The average amplitude of pulses for the peaking time setting 15,20 and 35 ns was 162,168 and $174 \mathrm{mV}$, respectively, the level of noise was $1.00,0.84$ and $0.80 \mathrm{mV}$, and the signal-to-noise ratio was 162, 200 and 218. The lowest noise of $0.80 \mathrm{mV}$ is observed for the longest peaking time ( $35 \mathrm{~ns})$. However, for this peaking time one can expect the biggest drift time uncer-

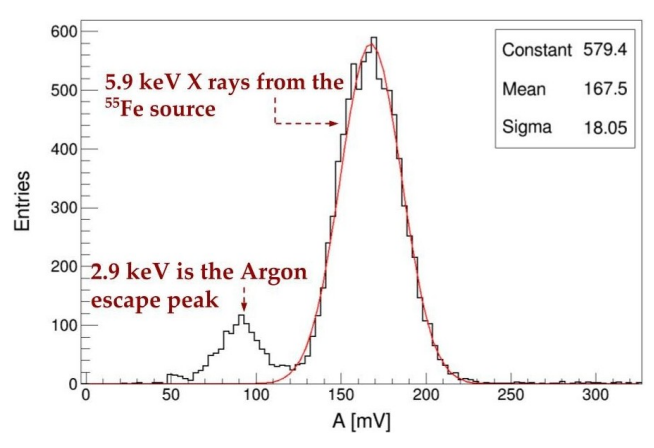

Fig. 1 Amplitude spectrum registered for the peaking time setting of $20 \mathrm{~ns}$ (black line) together with a gaussian function fit to the $5.9 \mathrm{keV}$ line.

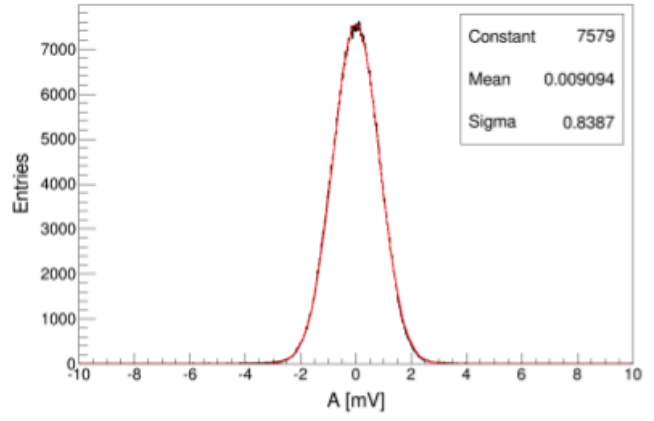

Fig. 2 Distribution of noise for the peaking time setting of $20 \mathrm{~ns}$ (black line) together with a fitted gaussian function (red line). tainty due to the time walk effect.

Therefore, we plan to find the optimal peaking time setting, allowing to obtain a low noise level and a satisfactory drift time resolution. We also conduct studies of additional shielding of the straws with extra layer of aluminized Mylar for further reduction of the noise.

Acknowledgements We acknowledge financial support by the Polish National Science Center grant agreement no. 2016/23/P/ST2/04066 and 2017/26/M/ST2/00600 POLONEZ, as well as by the Ministry of Science and Higher Education grant agreement no. 7150/E-338/M/2018.

\section{References}

1. Official website : https://panda.gsi.de/

2. J. Smyrski et al., JINST 12, C06032 (2017)

3. J. Smyrski et al.,JINST 13, P06009 (2018)

4. D. Przyborowski et al., JINST 11, P08009 (2016) 